\title{
Anesthetic considerations for a patient with situs inversus totalis undergoing cardiac surgery - A case report -
}

Received July 5, 2018

Revised 1st, August 28, 2018

2nd, September 12, 2018

Accepted September 12, 2018

\section{Corresponding author}

Jong Chan Kim, M.D., Ph.D. Department of Anesthesiology and Pain Medicine, CHA Bundang Medical Center, 59 Yatap-ro, Bundang-gu, Seongnam 13496, Korea

Tel: 82-31-780-5799

Fax: 82-31-780-5474

E-mail: aescula72@hanmail.net ORCID

https://orcid.org/0000-0001-7051-905X

\section{Chang-hoon Koo', Jae-Kwang Shim², Namhoon Kim', Yumin Ki', Junbeom Park', and Jong Chan Kim ${ }^{1}$}

Department of Anesthesiology and Pain Medicine, ${ }^{1} \mathrm{CHA}$ Bundang Medical Center, Seongnam, ${ }^{2}$ Severance Hospital, Yonsei University College of Medicine, Seoul, Korea

A 58-year-old male patient with situs inversus totalis, a rare congenital malformation characterized by all asymmetric organs being formed as the mirror images of their normal morphologies, underwent mitral valve repair due to mitral valve prolapse. This case was reported to suggest that anesthesiologists should thoroughly understand the anatomy of these types of patients before providing cardiac anesthesia that often requires advanced monitoring and rely on their accurate interpretation. Accordingly, a few key points will be discussed with emphasis on reversing lead placement during electrocardiogram monitoring, using the left internal jugular vein for pulmonary artery catheterization, and firmly comprehending mirror image heart morphology to better conduct transesophageal echocardiography.

Keywords: Anesthesia; Situs inversus totalis; Thoracic surgery.

Situs inversus totalis is a rare congenital anomaly in which all internal organs are arranged in the mirror image of their normal morphologies. Patients with situs inversus totalis are asymptomatic and usually lead normal lives, but the recognition of this condition is important to interpret patient symptoms of other conditions accurately and to avoid clinical or surgical mistakes. In addition, accurate understanding of the situs inversus totalis anatomy would be a prerequisite to an optimal anesthetic management for these patients, especially when advanced hemodynamic monitoring devices and their accurate interpretation are required as in cardiac surgery. This case was reported to share this medical team's experience of a 58-year-old male patient with situs inversus totalis who underwent mitral valve repair.

\section{CASE REPORT}

A 58-year-old male patient was scheduled to undergo elective mitral valve repair surgery to repair mitral valve prolapse (P3). The preoperative chest X-ray showed that his heart was directed to the right with mild cardiomegaly. The preoperative electrocardiogram (ECG) was conducted with chest leads in their normal positions and showed a negative $\mathrm{P}$-wave in the I and aVL leads and a positive P-wave in the aVR lead, a typical right-axis deviation pattern. Another ECG was then conducted with reversed lead placement positions, which produced normal results (Fig. 1). In his history, the patient stated that he had been diagnosed with situs inversus totalis 50 years ago and had lived without any symptoms of this condition for the duration of his life. The preoperative physical examination and evaluations did not produce any other significant findings except for mild dyspnea.

This is an Open Access article distributed under the terms of the Creative Commons Attribution Non-Commercial License (http://creativecommons.org/licenses/by-nc/4.0) which permits unrestricted non-commercial use, distribution, and reproduction in any medium, provided the original work is properly cited. 


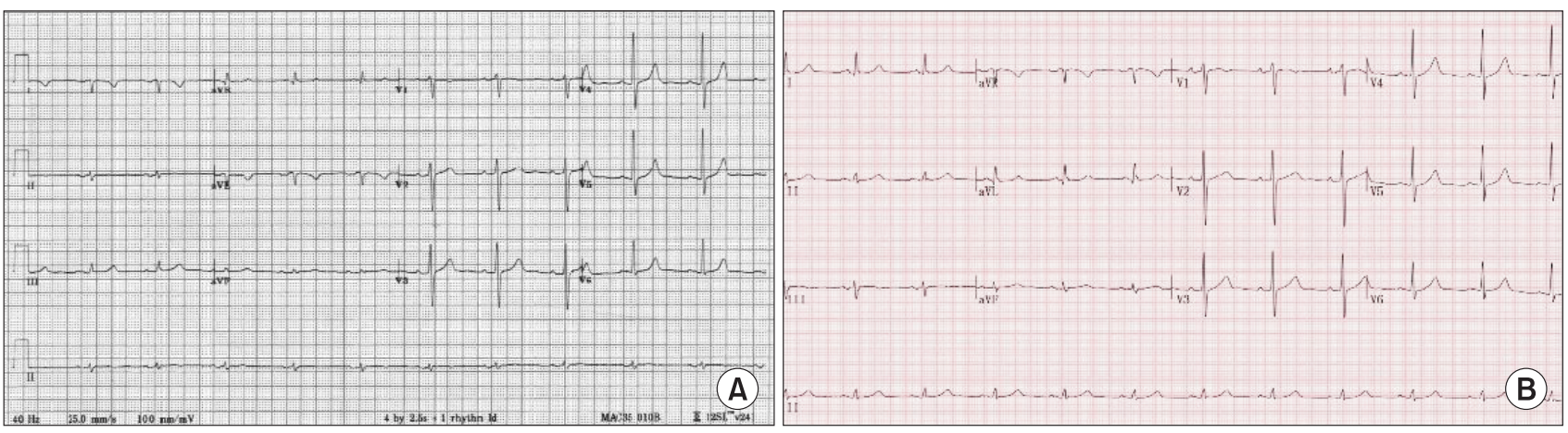

Fig. 1. (A) Echocardiogram with normal lead placement showing right axis deviation. (B) Normal echocardiogram with reverse lead placement. ECG: electrocardiogram.

Upon the patient's arrival in the operating room, ECG leads were applied in reverse to reduce confusions over the ECG findings and unnecessary treatments to correct abnormal ECG results produced by improperly placed leads. Other standard institutional monitoring methods were applied, including left radial arterial cannulation. Anesthetic induction was performed according to institutional guidelines and pulmonary artery catheterization was performed via the left internal jugular vein with MAC $^{\mathrm{TM}}$ Multi Access Catheter (ARROW international Inc., USA) to guarantee a direct approach to the right atrium without crossing the midline. The medical team attempted to maintain the right-curved shape of the pulmonary artery catheter when inserting it into the left internal jugular vein to facilitate the advancement of the catheter though the morphologic right heart. Catheterization was successfully performed uneventfully on the first attempt. The postoperative chest X-ray revealed that the right-curved shape of the pulmonary artery catheter was maintained as the mirror image of its normal shape (Fig. 2).

Intraoperative transesophageal echocardiography (TEE) was challenging. In this patient, a different angle was needed to acquire the desired views because of his atypical anatomy. Unlike the TEE images of normal hearts, the midesophageal four chamber and transgastric short axis views obtained near $0^{\circ}$ in normal hearts were obtained with the left ventricle on the left side of the TEE screen, which is the inverse of normal TEE views, so these views were obtained at a $180^{\circ}$ angle to reduce confusion (Fig. 3). The bicaval view, which is normally obtained at $110^{\circ}$, was obtained at $70^{\circ}$ while rotating the probe to the left in the direction of the right atrium (Fig. 4). The aortic valve short-axis view, normally found at $30^{\circ}-40^{\circ}$, was obtained at $140^{\circ}-150^{\circ}$ (Fig. 5). These views were best obtained at

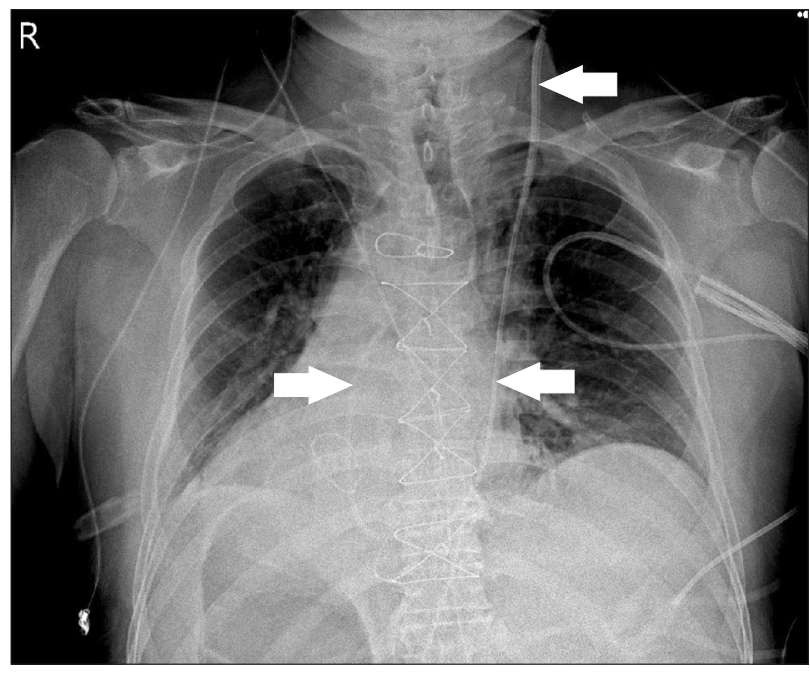

Fig. 2. Anteroposterior chest X-ray on postoperative day 1. Arrows show the right curved shape of pulmonary artery inserted directly via left internal jugular vein without crossing the midline.

an omniplane angle which was the difference of $180^{\circ}$ and the typical omniplane angle.

The operation was performed without incident. The patient was stable during and after the operation. The patient was transferred to the general ward after spending two days in the intensive care unit and discharged on the seventh day after the surgery without any complications.

\section{DISCUSSION}

Situs inversus totalis is a rare congenital condition in which all internal organs are the mirror images of their normal anatomies, occurring in $0.01-0.02 \%$ of people [1]. Situs inversus totalis is mainly an autosomal recessive disorder, but is sometimes related to $\mathrm{X}$-chromosomes. Human anatomical 

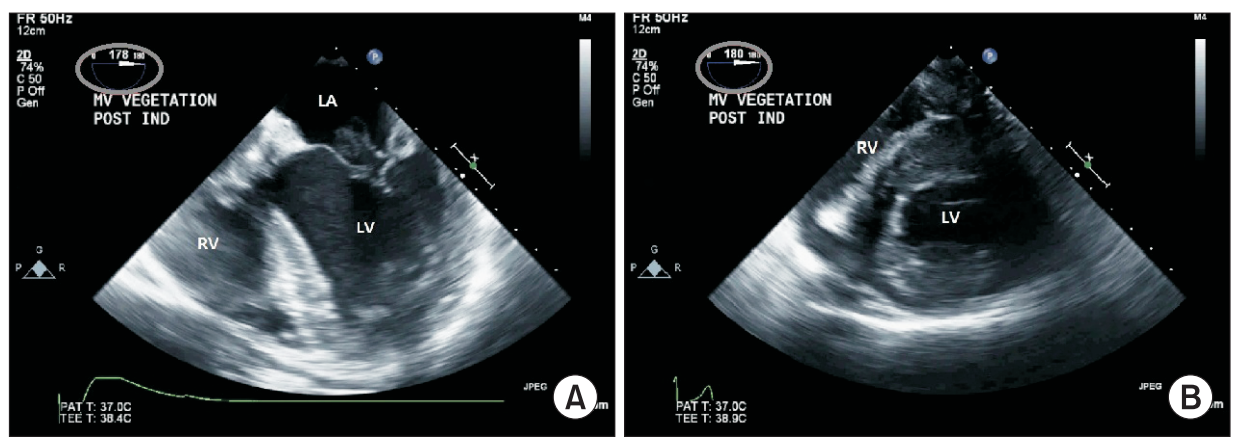

Fig. 3. (A) Transesophageal echocardiographic midesophageal four chamber view. (B) Transesophageal echocardiographic transgastric short axis view. These views were obtained at angle of $180^{\circ}$ (circle). LA: left atrium, LV: left ventricle, $\mathrm{RV}$ : right ventricle.

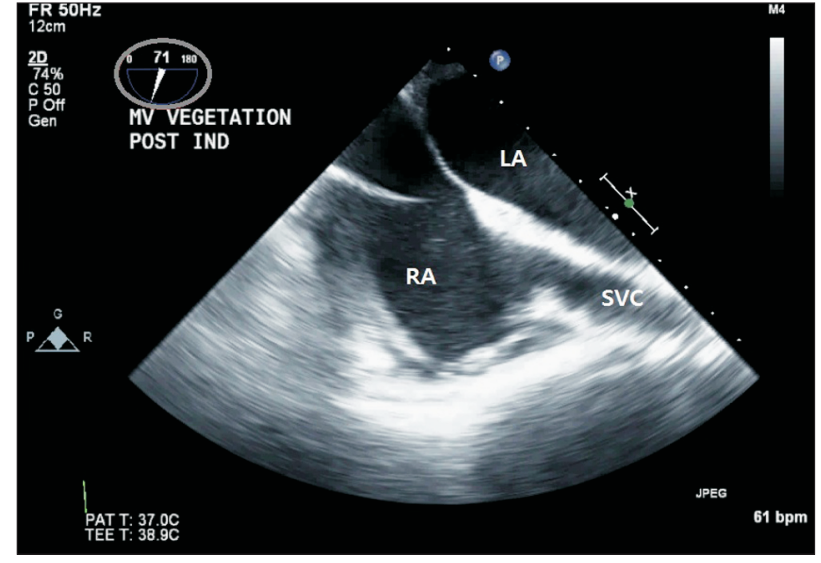

Fig. 4. Transesophageal echocardiographic bicaval view obtained at $70^{\circ}$ (circle). LA: left atrium, RA: right atrium, SVC: superior vena cava.

changes occur during embryogenesis. Although most patients with situs inversus totalis have normal life expectancies without any significant related morbidities, they should be meticulously evaluated because the condition may coexist with other congenital comorbidities in the cardiovascular, respiratory, and digestive systems [2]. When situs inversus totalis is combined with recurrent respiratory infections such as sinusitis and bronchiectasis in a patient, it is diagnosed as Kartagener's syndrome or primary ciliary dyskinesia. Repeated respiratory infections result from impaired mucociliary function [3]. In this case, the supplementation of humid oxygen, adequate patient's hydration and analgesia is mandatory to reduce any inspissation of secretion in the airway and prevent the compromised respiratory mechanics [4].

The recognition of anatomical changes and comorbidities by careful preoperative evaluation is necessary to prevent clinical mistakes and ensure that such patients receive appropriate treatment. It has been reported that thorough preoperative evaluations confirmed acute appendicitis in patients with situs inversus totalis suffering from left lower quadrant

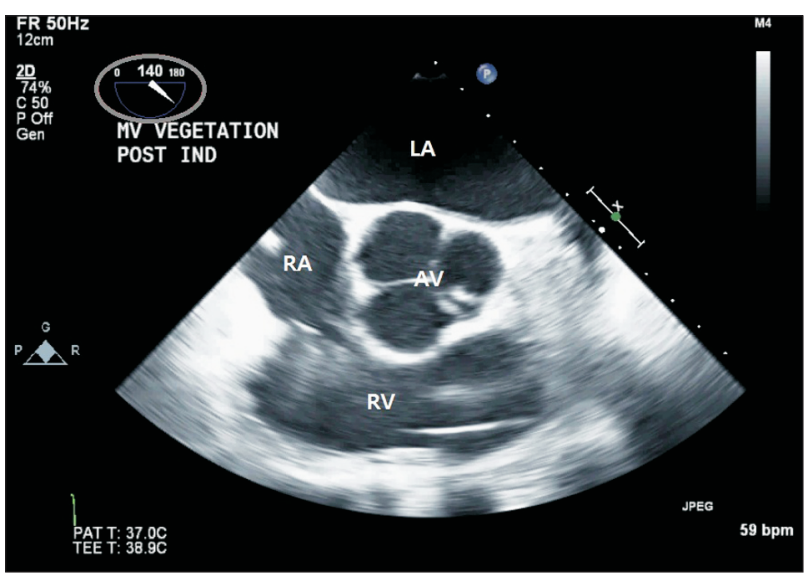

Fig. 5. Transesophageal echocardiographic aortic valve short-axis view obtained at $140^{\circ}$ (circle). AV: aortic valve, LA: left atrium, RA: right atrium, RV: right ventricle.

abdominal pain $[2,5]$. Moreover, accurate obtainment and interpretation of data from various monitoring devices would be challenging to the attending anesthesiologists, especially in case of cardiac surgery that requires additional monitors including the TEE.

As mentioned in the case report, the preoperative ECG with conventional lead placement showed right-axis deviation because of the anatomical variations resulting from situs inversus totalis. However, the ECG with reversed lead placement did not show any abnormalities, so this lead placement was maintained during and after surgery to prevent attending physicians from misinterpreting ECG results and giving unnecessary treatments. Likewise, physicians should remember that defibrillation pads should be applied to situs inversus totalis patients in reverse. Central venous catheterization via the left internal jugular vein has been recommended for patients with situs inversus totalis [3]. Accordingly, we chose the left internal jugular vein for pulmonary artery catheterization to avoid thoracic duct injury, which would be on the 
patient's right side, and guarantee a direct approach to the right atrium. The postoperative chest $\mathrm{X}$-ray showed that the pulmonary artery catheter cannulated through the left internal jugular vein followed a direct course to the superior vena cava instead of crossing the midline (Fig. 2).

In spite of a few recommandation in TEE practice in patients with situs inversus totalis [6], it may be difficult for anesthesiologists to conduct TEE monitoring of situs inversus totalis patients during surgery, so they must be vigilant when examining cardiovascular structures such as the ventricles, atria, and aorta. Views obtained near $0^{\circ}$, including the midesophageal four chamber and trans-gastric short axis views are mirror images of the normal views (Fig. 3). Other standard views can be obtained at an omniplane angle of $180^{\circ}$ minus the typical omniplane angle. For instance, the bicaval view, which is normally obtained at $110^{\circ}$, can be obtained at $70^{\circ}$ while rotating the probe to the left because the right atrium is located to the left (Fig. 4). The aortic valve short-axis view, normally found at $30^{\circ}-40^{\circ}$, can be obtained at $140^{\circ}-150^{\circ}$ (Fig. 5). In order to reduce confusion regarding the omniplane angle adjustment, clinicians may prefer to acquire these views via right-left invert of the obtained images through the TEE machine. Still, the left-right maneuvering directions of the TEE probe need to be opposite, which can be quiet challenging to the attending physician.

Patients with situs inversus totalis are difficult to anesthetize and monitor while under anesthesia. However, these patients can be managed more safely with careful preoperative planning and increased vigilance. This case was reported to describe a few key considerations for the proper anesthetic management of a situs inversus totalis patient during cardiac surgery with special emphasis to monitoring devices including the TEE.

\section{CONFLICTS OF INTEREST}

No potential conflict of interest relevant to this article was reported.

\section{ORCID}

Chang-hoon Koo: https://orcid.org/0000-0001-8567-5514

Jae-Kwang Shim: https://orcid.org/0000-0001-9093-9692

Namhoon Kim: https://orcid.org/0000-0002-8204-5979

Yumin Ki: https://orcid.org/0000-0002-3922-5798

Junbeom Park: https://orcid.org/0000-0001-8193-4822

\section{REFERENCES}

1. Garg N, Agarwal BL, Modi N, Radhakrishnan S, Sinha N. Dextrocardia: an analysis of cardiac structures in 125 patients. Int J Cardiol 2003; 88: 143-55.

2. Channabasappa SM, Mohan HS, Sarma J. A patient with situs inversus totalis presenting for emergency laparoscopic appendectomy: consideration for safe anesthetic management. Anesth Essays Res 2013; 7: 127-9.

3. Bush A, Chodhari R, Collins N, Copeland F, Hall P, Harcourt J, et al. Primary ciliary dyskinesia: current state of the art. Arch Dis Child 2007; 92: 1136-40.

4. Garg R, Goila A, Sood R, Pawar M, Borthakur B. Perioperative anesthetic management of a patient with biliary atresia, situs inversus totalis, and kartegener syndrome for hepatobiliary surgery. J Anaesthesiol Clin Pharmacol 2011; 27: 256-8.

5. Koç A, Sönmez Y, Balaban O. Anaesthetic management for appendectomy in a patient with situs inversus totalis. Turk J Anaesthesiol Reanim 2016; 44: 105-7.

6. Raut MS, Maheshwari A, Shad S, Rachna G. How standard transesophageal echocardiography views change with dextrocardia. Ann Card Anaesth 2013; 16: 218-20. 